\title{
Urinary levels of 6-sulphatoxymelatonin and their associations with sleep disorders and behavioural impairments in children with autism spectrum disorder
}

\author{
Babinska $\mathrm{K}^{1}$, Siklenkova $\mathrm{L}^{1}$, Stebelova $\mathrm{K}^{2}$, Waczulikova $\mathrm{I}^{3}$, Celusakova $\mathrm{H}^{1}$, Vidosovicova $\mathrm{M}^{1}$, \\ Bartakovicova $\mathrm{K}^{1}$, Szapuova $\mathrm{Z}^{1}$, Kemenyova $\mathrm{P}^{1}$
}

Academic Centre for Autism Research, Institute of Physiology, Comenius University, Faculty of Medicine, Bratislava, Slovakia. katarina.babinska@fmed.uniba.sk

\begin{abstract}
BACKGROUND: Autism spectrum disorder (ASD) is a neurodevelopmental condition associated with sleep disturbances that may result from abnormalities in melatonin production. The correlations of melatonin levels with the severity of sleep disorder and/or severity of ASD were reported.

OBJECTIVES: To evaluate urinary levels of the melatonin metabolite, 6-sulphatoxymelatonin (aMT6s), in children with ASD, and their associations with sleep abnormalities and behavioural impairments. METHODS: Study involved 77 children with ASD and 84 controls aged 2.5-15.5 years. Sleep disorders were assessed by Children's Sleep Habits Questionnaire. Morning and afternoon levels of aMT6s were determined by radioimmunoassay method. Urinary creatinine levels were assessed by an enzymatic method.

RESULTS: The urinary aMT6s/creatinine values indicate that the night-time melatonin levels are significantly lower in ASD than in controls, but there are no significant differences in the daytime levels. In the ASD group, on average, a 6.8-fold difference between night-time and daytime values of urinary aMT6s/creatinine was found, whereas for the controls a 12.5-fold difference was observed, indicating a lower night-time increase in melatonin levels. In ASD group, the difference in night-time-daytime aMT6s/creatinine value correlated with some types of sleep problems, but not with the severity of ASD.

CONCLUSION: The results indicate that in ASD there are differences in the patterns of melatonin secretion that may be associated with sleep impairment (Tab. 4, Fig. 2, Ref. 28). Text in PDF www.elis.sk.

KEY WORDS: autism spectrum disorder, sleep disturbances, 6-sulphatoxymelatonin.
\end{abstract}

\section{Introduction}

Autism spectrum disorder (ASD) is a neurodevelopmental disorder characterized by social and communications impairments as well as by restrictive and repetitive patterns of behaviour and interests. It is a lifelong disability affecting about $1 \%$ of children (1). The exact pathomechanism of ASD is not explained $(2,3)$. A variety of comorbidities are associated with ASD, including gastrointestinal disorders, epilepsy, or psychiatric illnesses, with sleep impairments being one of the most common ones (4). The

${ }^{1}$ Academic Centre for Autism Research, Institute of Physiology, Comenius University, Faculty of Medicine, Bratislava, Slovakia, ${ }^{2}$ Department of Animal Physiology and Etology, Comenius University, Faculty of Natural Sciences, Bratislava, Slovakia, and ${ }^{3}$ Department of Nuclear Physics and Biophysics, Comenius University, Faculty of Mathematics, Physics and Informatics, Bratislava, Slovakia

Address for correspondence: $\mathrm{K}$. Babinská, $\mathrm{MD}$, $\mathrm{PhD}$, Comenius University, Faculty of Medicine, Institute of Physiology, Sasinkova 2, SK-813 72 Bratislava, Slovakia.

Phone: +421.2 .90119523$

Acknowledgements: Supported by grants APVV 15-0085, APVV 150045, APVV 18-0174, VEGA 1/0141/17. prevalence of sleep disturbances in children with ASD is reported to be $50-80 \%$, compared to $9-50 \%$ in typically developing children (5). In particular, short sleep duration, low sleep quality/efficiency, circadian sleep desynchronisation and/or delayed sleep are the most commonly presented sleep disturbances (6).

There is evidence that individuals with ASD who suffer from sleep impairments may present with increased severity of ASD core symptoms (6). In a study, the sleep onset delay was linked to stereotyped behaviours and impairments of social interaction, while parasomnia has been associated with communication problems and increase in stereotyped behaviours (7).

Melatonin, primarily produced by the pineal gland, is one of the major regulators of the sleep cycle (8). Its secretion exhibits diurnal variation: it is low during the day since the pineal melatonin production is powerfully suppressed by light exposure. During the night, melatonin concentrations increase several times with a peak in the middle of the night (9). Several studies revealed that a proportion of individuals with ASD have lower levels of melatonin (5), as well as that deviations in biorhythm of melatonin secretion were observed (6). Abnormalities in melatonin production have been associated with the severity of ASD impairments (10). 


\section{9-855}

The main metabolite of melatonin is 6-sulphatoxymelatonin (aMT6s) which is excreted into urine. Urinary aMT6s excretion rates have been shown to correlate with serum melatonin levels (11), thus, urinary aMT6s concentrations are widely used as a convenient biomarker of melatonin secretion (9). It has been found that single morning urinary sample reflects well the overnight urinary aMT6s excretion rates (12).

The objective of the study was to examine the levels of aMT6s in spot urine samples of children with ASD as an indicator of serum melatonin levels. In order to examine the daily variation, a urine sample was collected in the morning as an indicator of the night-time plasma melatonin concentration, and another one was obtained during the day, reflecting the daytime melatonin level. In addition, we aimed to analyse the associations of aMT6s with sleep disorders and severity of ASD symptoms.

\section{Material and methods}

\section{Sample}

The case-control study involved 77 children with ASD recruited at the Academic Centre for Autism Research. Individuals with associated genetic disorders and severe psychiatric or neurological comorbidities were excluded from the survey. The control sample involved 84 age-matched subjects with typical neurodevelopment recruited from schools and preschool facilities. None of the individuals were reported to be using melatonin supplements. The main characteristics of the sample are shown in Table 1.

\section{Behavioral assessment}

The diagnosis of ASD was determined according to ICD-10 by trained examiners. The diagnostic tools involved included the Autism Diagnostic Observation Schedule - Second Edition (ADOS2) (13), and Autism Diagnostic Interview-Revised (ADI-R) (14).

ADI-R was evaluated in areas of qualitative abnormalities in social interaction impairments domain (ADI-R Domain A), communication and language impairments domain (ADI-R Domain B), as well as in repetitive and stereotyped behaviour domain (ADI-R Domain C). Because of possible differences in the raw score resulting from a different number of items evaluated according to age, the ADI-R scores were transformed. The final score in each ADI-R domain was calculated as a ratio between the individual's raw score and maximum possible score that the individual could achieve (i.e. sum of maximal scores of all items evaluated). Thus, the transformed values are expressed in percent $(\%)$.

ADOS-2 was evaluated in domains of social effect (SE \%) and restricted and repetitive behaviour (RRB \%). The children were examined by different modules of ADOS- 2 according to the level of speech achieved. ADOS-2 Calibrated Severity Score (ADOS-2

Tab. 1. Characteristics of the study groups.

\begin{tabular}{lcccc}
\hline Characteristics & Levels & ASD & Controls & $p$ \\
\hline Total count & & 77 & 84 & \\
\hline Sex & boys & $67(87 \%)$ & $62(74 \%)$ & \multirow{2}{*}{0.032} \\
\hline Age (years) & median (range) & $4.9(2.6-14.9)$ & $5.7(2.5-15.5)$ & 0.201 \\
\hline
\end{tabular}

CSS) was used, allowing to compare the severity of ASD symptoms in different modules of ADOS-2 (15).

The ASD diagnosis was made based on clinical evaluation, while all children enrolled in the study had to meet the criteria for ASD on both ASD diagnostic tools.

\section{Assessment of sleep habits}

Children's Sleep Habits Questionnaire (CSHQ) was used to quantify sleep problems (16). The CSHQ is a scale based on parent-reported items assessing main sleep domains. CSHQ total score of 41 is the clinical cut-off for identification of probable sleep problems while higher scores indicate a higher occurrence of sleep problems. The components of CSHQ can be grouped into eight subscales representing common domains of sleep disorders in children. The CSHQ has been validated in preschool and schoolchildren and it is widely used for screening sleep disorders also in ASD (6).

\section{Urine collection and analysis}

Parents collected the first morning sample at home after the child had woken up. The afternoon sample was collected at lunchtime or in early afternoon. The samples were frozen and stored at $-20{ }^{\circ} \mathrm{C}$ until analysis.

The urinary levels of aMT6s were determined by the radioimmunoassay (RIA) method. We used a commercially available kit (Stockgrand LTD., Guildford, UK) and the assay was performed according to manufacturer's instructions using the method of Arendt et al. (12) adapted for iodinated aMT6s (17). The samples were measured within 5 assays. The mean intraassay coefficient was $12.9 \%$. The mean interassay coefficient was $8.0 \%, 7.7 \%$ and $13.2 \%$ for low, medium and high-quality control sample, respectively. The level of creatinine was determined using an enzymatic method for quantitative in vitro determination of creatinine in human serum, plasma and urine (Erba Lachema, CZ) according to manufacturer's instructions.

\section{Statistical analysis}

The demographic and clinical characteristics were analysed using descriptive statistics. Two-sample t-test or alternatively MannWhitney test was used to test for between-group differences. To assess daytime differences in standardized aMT6s, the data were logarithmically transformed with base 2 to ensure the linearity of response. Categorical variables are presented as counts and relative frequencies and differences between two categorical variables were tested by chi-square or binomial tests. The Spearman's rank correlation coefficients were used to determine the associations between selected variables.

Statistical analyses were performed using StatsDirect 3.0.191 software (Stats Direct Ltd., Cheshire, UK) and Statistica 13.1 software (TIBCO Software Inc., US). All p-values were considered significant at a two-tailed $\mathrm{p}<0.05$.

\section{Ethics statement}

The study protocol was approved by the Ethics Committee of the University Hospital and Comenius University Faculty of 
Tab. 2. Bivariable analysis of aMT6s, creatinine and aMT6s/creatinine levels in the morning and afternoon urine samples of children with ASD and controls.

\begin{tabular}{lcccc}
\hline Characteristics & ASD & Controls & Difference ASD-C & p \\
\hline M - aMT6s, ng/mL & $55.3(38.6-119.9)$ & $76.0(45.2-112.2)$ & -20.71 & 0.259 \\
M - creatinine, mg/mL & $0.72(0.44-1.14)$ & $0.52(0.34-0.71)$ & 0.20 & 0.001 \\
M - aMT6s/creatinine, ng/mg & $92.02(58.38-149.64)$ & $149.72(90.38-223.33)$ & -57.70 & 3.15 \\
A - aMT6s, ng/mL & $6.98(2.76-14.59)$ & $3.83(1.25-8.39)$ & 0.001 \\
A - creatinine, $\mathrm{mg} / \mathrm{mL}$ & $0.57(0.31-1.14)$ & $0.34(0.14-0.66)$ & 0.93 & 0.002 \\
A - aMT6s/creatinine, ng/mg & $11.37(5.44-22.21)$ & $10.39(4.56-20.54)$ & 0.001 & 0.221 \\
\hline
\end{tabular}

Data are expressed as medians and lower - upper quartiles (Q1-Q3), M - morning values, $\mathrm{A}$ - afternoon values, $\mathrm{p}$ - statistical significance

Medicine and University Hospital in Bratislava, Slovakia. Written informed consent was obtained from caregivers of all participating children. The study was in accordance with the Declaration of Helsinki.

\section{Results}

Concentrations of aMT6s, creatinine and aMT6s/creatinine in the morning (M) and afternoon (A) urinary samples are displayed in Table 2 . The urinary creatinine concentration was significantly higher and displayed a wider variability in our group with ASD.
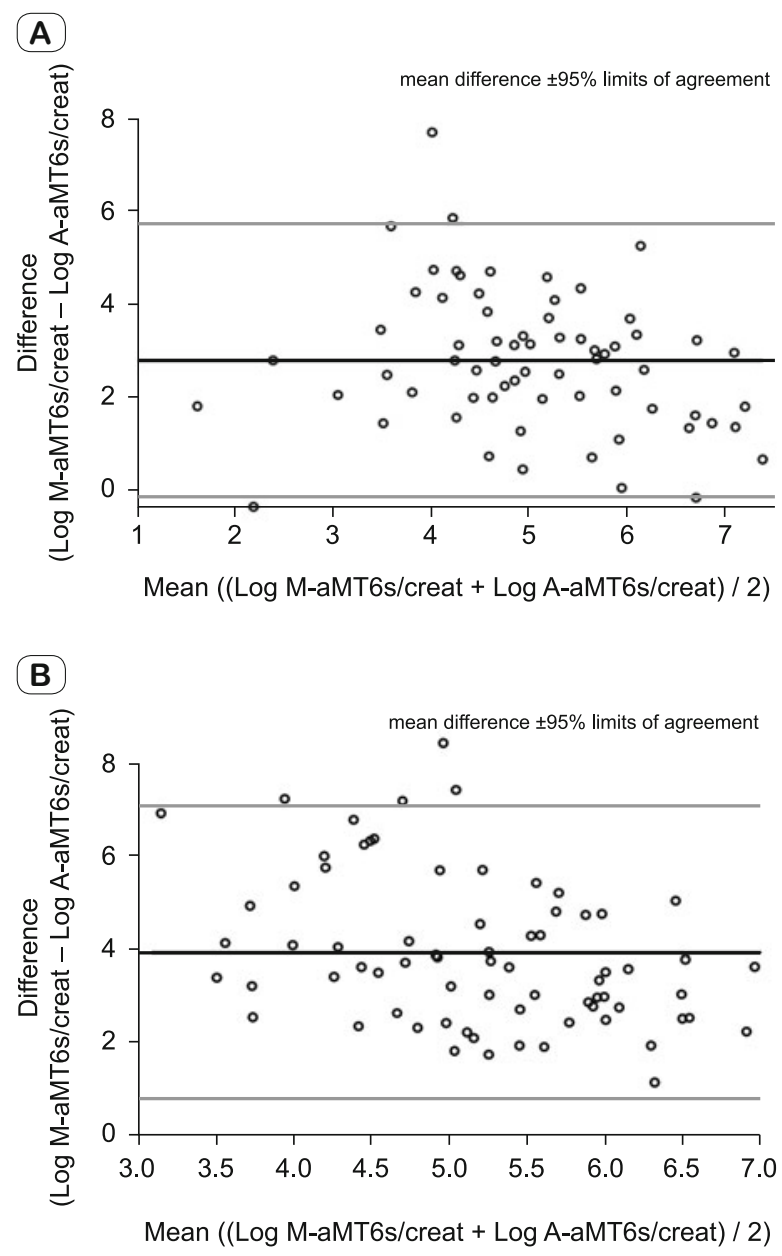

Fig. 1. Bland-Altman plot analysing the agreement between morning (M) and afternoon (A) levels of aMT6/creatinine.
Tab. 3. The comparison of children's sleep habits questionnaire (CSHQ) scores between the ASD and control groups.

\begin{tabular}{llccc}
\hline CSHQ & Levels & $\begin{array}{c}\text { ASD } \\
(\mathrm{n}=67)\end{array}$ & $\begin{array}{c}\text { Controls } \\
(\mathrm{n}=83)\end{array}$ & $\mathrm{p}$ \\
\hline Total score & mean \pm SD & $47.4 \pm 8.77$ & $42.9 \pm 7.01$ & 0.0008 \\
\hline Sleep disorder & yes & 53 & 54 & $0.0262^{\mathrm{a}}$ \\
\hline no & 12 & 29 & \\
\hline Bedtime resistance & & $10.5 \pm 3.46$ & $8.4 \pm 2.55$ & 0.0001 \\
\hline Sleep onset delay & Rarely & 36 & 58 & \\
& Sometimes & 15 & 17 & $0.0201^{\mathrm{b}}$ \\
& Usually & 14 & 7 & \\
\hline Sleep duration & & $4.1 \pm 1.58$ & $3.3 \pm 0.75$ & 0.0002 \\
\hline Sleep anxiety & & $6.6 \pm 2.20$ & $5.8 \pm 1.92$ & 0.0260 \\
\hline Night waking & & $4.3 \pm 1.61$ & $3.8 \pm 1.28$ & 0.0527 \\
\hline Parasomnia & & $9.0 \pm 2.07$ & $8.3 \pm 1.74$ & 0.0157 \\
\hline Sleep breathing disorder & $3.32 \pm 0.77$ & $3.31 \pm 0.62$ & 0.9319 \\
\hline Daytime sleepiness & & $11.8 \pm 3.59$ & $12.0 \pm 3.43$ & 0.7024 \\
\hline $\begin{array}{l}\text { ASD }- \text { autism spectrum disorder, SD }- \text { standard deviation, }{ }^{*} \text { chi-square test, **test } \\
\text { of linear trend }\end{array}$ & & &
\end{tabular}

Since this factor influences the urinary concentration of biomarkers (18), creatinine-standardised aMT6s (aMT6s/creatinine) values were chosen as the primary biochemical marker for our analyses.

The morning values of aMT6s/creatinine were significantly lower in the group of children with ASD (median 92.02; lowerupper quartiles 58.38-149.64) than in the controls (median 149.72; lower-upper quartiles $90.38-223.33$; $<<0.001$ ). Since the morning values of aMT6s/creatinine reflect the melatonin production dur-

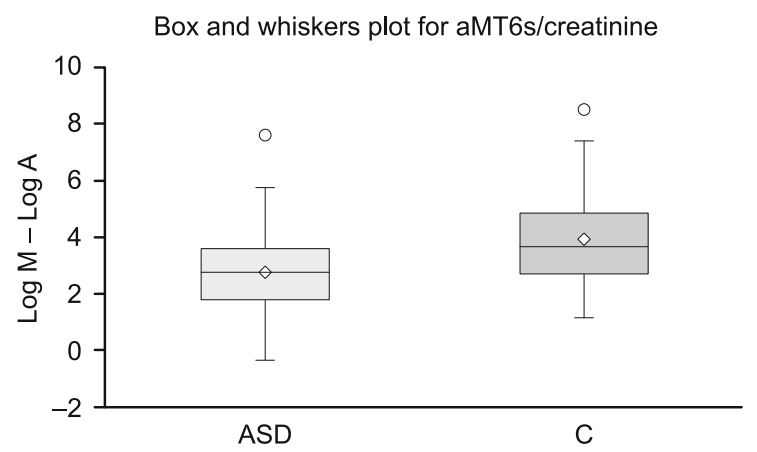

Fig. 2. Differences between $\log$-transformed morning $(\log M)$ and afternoon $(\log A)$ values of aMT6/creatinine levels in children with autism spectrum disorders (ASD) and controls (C). Medians are depicted as lines within the boxes and the respective mean with a diamond sign. The difference was statistically significant $(p<0.001)$. 
849-855

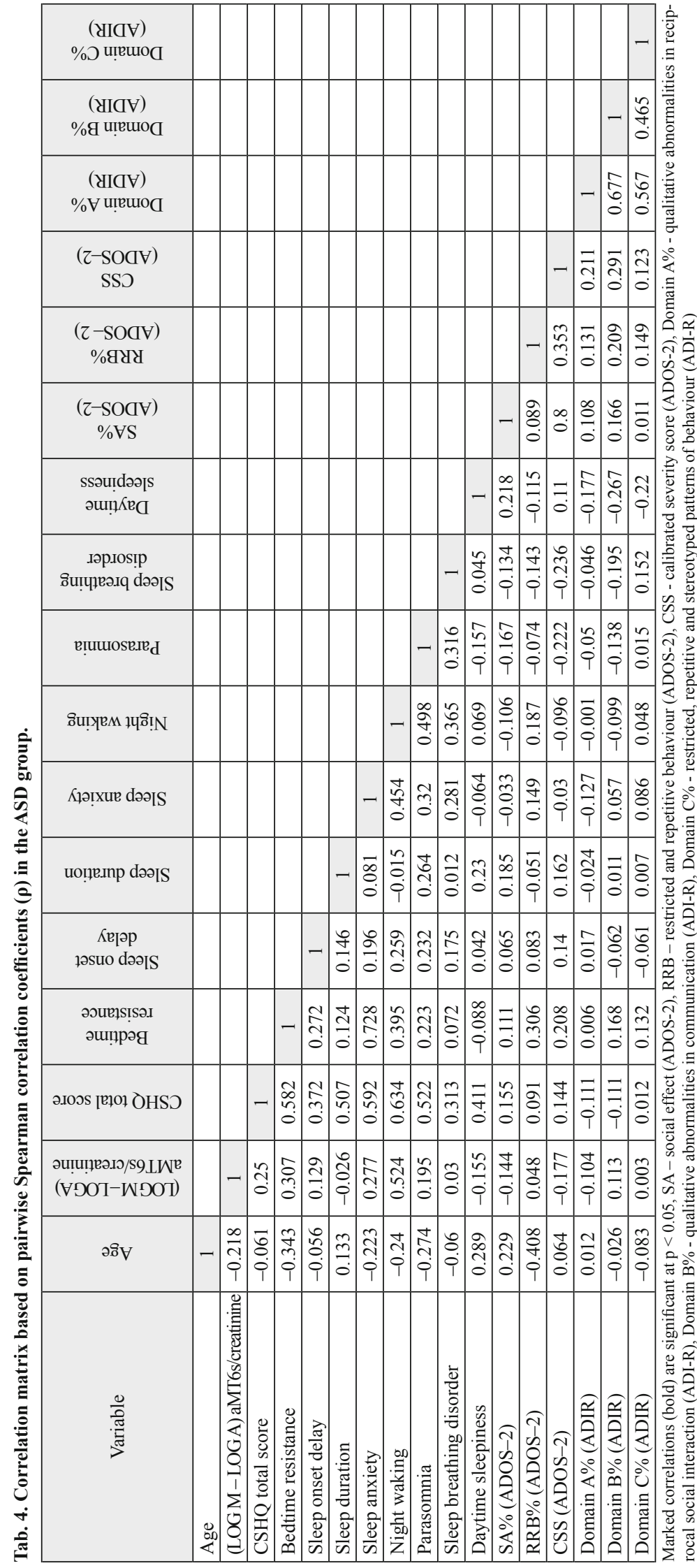

ing the night, our results indicate lower night-time levels of melatonin in the group with ASD. The values of aMT6s/creatinine in the afternoon sample displayed no significant difference between ASD (median 11.37, lower-upper quartile 5.44-22.21) and control (median 10.39; lower-upper quartile 4.56-20.54) groups $(\mathrm{p}=0.221)$, which indicates that the daytime melatonin levels may not differ between the groups.

The group averages do not reflect individual daily changes in aMT6s levels. In addition, it has been shown that aMT6s excretion may vary considerably between subjects (12). Therefore, for all subjects who provided both morning and afternoon samples of urine (67 for the ASD group; 73 for the control group) we evaluated the statistical differences between the morning and afternoon aMT6s concentrations (M-A difference) as an indicator of individual variation in daytime versus night-time melatonin levels. All children from both ASD and control groups displayed a decrease in the aMT6s/ creatinine value in the afternoon urine, with an exception of one individual with ASD in whom a mild increase was found $(+4.2 \mathrm{ng} / \mathrm{mg})$. The agreement between morning (M) and afternoon (A) aMT6/ creatinine levels are presented in Figure 1.

The afternoon levels were systematically lower than the morning ones in both, ASD (Fig. 1A) and control (Fig. 1B) groups, and resulted in positive global mean difference lines that were significantly different from the zero difference on the $\log 2$-scale (both $\mathrm{p}<0.001$ ). The mean difference in ASD group amounted to 2.8 with $95 \%$ CI: 2.4 to 3.1, and, that in control group reached 3.6 with $95 \%$ CI: 3.6 to 4.3 .

Pairwise differences between the morning and afternoon log-transformed values were statistically significant in both ASD and control groups $(\mathrm{p}<$ 0.001). The mean difference in ASD group (Fig. 1A) calculated from individual differences was significantly lower when compared to that in the control group (Fig. 1B). The graphical presentation in Figure 2 allows for better visual comparison. Since the constant additive change on the log scale is a constant multiplicative change on the original scale, we can state that the afternoon values of urinary aMT6s/creatinine in ASD were, on average, 6.8 times lower than the morning ones ( $95 \%$ confidence interval from 5.3 to 8.7 ), and for controls, 12.5 times lower with $95 \%$ CI from 11.8 to 19.9 .

The data on sleep habits of children with ASD and typically developing children are presented in Table 3. In comparison to the controls, a significantly higher proportion of children with ASD reported sleep problems $(65.0 \%$ vs $81.5 \%$; $=0.026)$. In 
ASD, the sleep problems occurred with higher frequency as indicated by the higher average values of the CSHQ total score. As to different types of sleep disorders, in the group with ASD, there were significantly higher CSHQ scores for bedtime resistance, sleep duration, sleep anxiety, and parasomnias, showing higher frequency of these sleep impairments in ASD than in controls. Also, the sleep onset delay in ASD occurred with a significantly higher frequency as shown by a larger proportion of individuals who reported this problem to present "usually", whereas in the control group, a rather rare occurrence predominated.

We further explored pairwise linear correlations (Tab. 4). Among those, we were focused on the strength of association between the morning and afternoon aMT6/creatinine difference (denoted as LOG M-LOG A aMT6s/creatinine) with sleep disorders and behavioural characteristics of the children with ASD.

The value of LOG M-LOG A aMT6s/creatinine significantly correlated with bedtime resistance $(\rho=0.307)$, sleep anxiety $(\rho=$ 0.277 ) and night waking $(\rho=0.524)$, but not with global severity of the sleep disorder assessed by the CSHQ total score. We have not found any significant correlations between LOG M-LOG A aMT6s/creatinine and severity of ASD core symptoms represented either by the ADOS-2 scores, namely calibrated severity score (ADOS-2 CSS), social effect, restricted and repetitive behaviour, or ADI-R domains, namely those of social interaction impairments, communication and language impairments, and repetitive and stereotyped behaviours.

Only inconsistent correlations between sleep disorder severity and autism severity were observed: the bedtime resistance significantly correlated with repetitive and restrictive behaviour $(\rho=0.306)$, while daytime sleepiness negatively correlated with communication and language impairment domain $(\rho=-0.267)$.

\section{Discussion}

A considerable number of studies provide evidence about higher prevalence of sleep disturbances in children with ASD (6), while most of the surveys are based on sleep questionnaires. Also, a recent meta-analysis of studies in which objective measures such as actigraphy or the "golden standard" method - polysomnography, were used, has confirmed a shorter total sleep time, longer sleep latency and lower sleep efficiency in ASD (19). Correspondingly, in our study, sleep disorders occurred with a significantly higher frequency in the group with $\mathrm{ASD}$, and with prevalence rates similar to those in other studies (6).

According to current evidence, the sleep disturbances in ASD have a multifactorial origin, but their exact causes are not fully explained. It is hypothesised that the underlying biological and behavioural specifics of ASD predispose to a higher sensitivity to extrinsic and intrinsic factors that threaten sleep, thus making the individuals more vulnerable to sleep problems. The abnormalities in pineal production of melatonin, the key player in circadian rhythm regulation, seem to be one of the intrinsic causes of sleep disturbances in ASD (20).

Studies investigating melatonin release patterns in ASD revealed differences in comparison to non-ASD subjects $(6,10)$.
The 24-hour measurements have shown a significantly lower level of serum melatonin, as well as a smaller nocturnal increase in the levels in children with $\operatorname{ASD}(21,22)$. Some studies revealed also deviations in daytime melatonin levels in ASD (10). Measurements of urinary aMT6s as an indirect marker of melatonin status, documented lower overnight aTM6s excretion rates in ASD (23-25). In our study, standardised aMT6s/creatinine was used as a marker of melatonin levels, and our results indicate lower night-time levels and smaller circadian variation of melatonin concentration in ASD. In accordance with the above-mentioned studies, our results support the hypothesis about melatonin abnormalities in ASD.

On the other hand, there are studies that failed to observe significant differences in melatonin levels in ASD. In general, the studies performed to date are heterogeneous in their design, time of sample collection, or type of the biological material used, and it has been suggested that additional research is needed to shed more light on patterns of melatonin release in ASD and their association with sleep problems (10).

Some recent studies aimed at genetics of melatonin found variations of genes regulating the synthesis, metabolism, and mechanisms of action of melatonin in ASD. They indicate that abnormalities in the expression of genes involved in melatonin pathways may be responsible for low melatonin levels and circadian sleep disturbances in subjects with $\operatorname{ASD}(6,10)$. Lower melatonin levels were observed in healthy mothers of children with ASD, thus suggesting a genetic origin of melatonin abnormalities (26). Since embryo and foetus do not produce melatonin, they are dependent on its maternal supply. At the same time, melatonin affects the brain development, neurogenesis and synaptogenesis. Therefore, low maternal melatonin levels may adversely affect these processes. Genetic studies also support the hypothesis that low melatonin is not a consequence, but a part of pathomechanisms of ASD (26).

Melatonin plays a well-established role in the regulation of circadian rhythm, however, it has a variety of other effects $(8,27)$. The most commonly cited are antiinflammatory, antioxidant and immunomodulatory properties; it also enhances mitochondrial function and displays a neuroprotective effect. These processes may be interfered by abnormalities in melatonin levels. Thus, melatonin may be involved in pathophysiology of ASD not only by causing impairments of the sleep-wake cycle, but also by other mechanisms (10). In fact, mitochondrial and immune dysfunction, as well as oxidative stress, have been shown to play a role in pathomechanisms of ASD (4).

A few studies that investigated correlations of melatonin levels with sleep problems in ASD found either negative or no correlations (6). In our study, the difference between daytime and night-time creatinine-standardised aMT6s values positively correlated with some types of sleep impairments. In other words, the more "normal" the pattern of melatonin secretion, the higher the probability of more severe sleep problem. To explain this finding, further investigation is needed, while one explanation may lie in the existence of specific subtypes of melatonin production. In a recent study (28), various melatonin production and excretion patterns were found in healthy children with sleep disorders, which highlights the importance of analysing specific alterations 


\section{9-855}

of aMT secretion in each sleep disorder. Also, some studies have shown that children with ASD may show sleeplessness with two distinct circadian deviations (6).

Tordjman et al., who used in their studies the same methods of behavioural assessment as we did in our study (ADOS-2, ADI-R), have found a negative correlation between nocturnal secretion of aTM6s and severity of some of the core symptoms in adolescents with $\operatorname{ASD}(23,24)$. In our study, these correlations were not observed. One of the possible reasons for that could be the difference in age of the study participants.

Sleep disturbances have a negative impact on the core symptoms of ASD, and they may also adversely affect the adaptive behaviour and exacerbate the challenging behaviours such as aggression and self-injury. They often interfere with therapeutic interventions and education of the child with ASD, as well as with general everyday life and well-being of the family (4). Additionally, abnormal circadian rhythms and impairments in sleep quality have been shown to be associated with higher risks of cardiovascular, metabolic diseases, and mortality (8), which are growing problems in people with ASD.

Melatonin in form of supplements is commonly used in the treatment of sleep disorders, also in individuals with ASD. Several studies have shown positive effects of melatonin administration on sleep initiation and duration in children with ASD (9). However, there is still a non-negligible proportion of children already taking melatonin and continuing to have sleep problems. It was suggested that this may be partly due to different types of alterations in melatonin production, since in some children with sleep impairments a normal pattern was observed, while some children displayed melatonin hyperproduction, low production or absence of circadian variation (28).

There is evidence that melatonin has positive effects also on anxiety, gastrointestinal dysfunction, and sensory processing that are frequent comorbidities of ASD with adverse consequences on sleep. It seems that melatonin could at least partially exert some of its positive effects on sleep in ASD by acting on these alternative routes (10).

This was the first survey dealing with sleep disturbances in children with ASD in Slovakia aimed at investigating their melatonin status. The study is limited by the fact that some possibly confounding factors of melatonin levels $(8,12)$ such as environmental exposure to light, seasonality, diet and exercise or stage of puberty were not controlled. The individuals in the control sample were not screened for possible ASD symptoms by standard tools; we relied only on parental reports of their typical neurodevelopment.

In conclusion, in a study involving children aged 2.5-15.5 years we have shown that the prevalence of sleep disorders was higher in the group with ASD. The higher frequency of bedtime resistance was more likely to be associated with higher ADI-R scores (i.e. more severe presentation) in the communication and language impairments domain. The results show deviations in melatonin status in children with ASD. The urinary values of aMT6s/creatinine found in our study indicate a lower melatonin production during the night and a smaller night-time increase in melatonin levels in individuals with ASD. Alterations in melatonin levels reinforce the idea about its possible role of a biomarker in ASD. The high prevalence of sleep disorders suggests that sleep investigation should be part of multidisciplinary clinical evaluation of a child with ASD.

\section{References}

1. American Psychiatric Association. Diagnostic and Statistical Manual of Mental Disorders. American Psychiatric Association, 2013.

2. Matelski L, Van de Water J. Risk factors in autism: Thinking outside the brain. J Autoimmun 2016; 67: 1-7.

3. Važan R, Belica I, Ostatníková D. Etiopathogenesis of Autism. Lek Obzor (Med Horizon) 2018; 67 (7-8): 234-238.

4. Frye RE, Rossignol DA. Identification and Treatment of Pathophysiological Comorbidities of Autism Spectrum Disorder to Achieve Optimal Outcomes. Clin Med Insights Pediatr 2016; 10: 43-56.

5. Geoffray M-M, Nicolas A, Speranza M, Georgieff N. Are circadian rhythms new pathways to understand Autism Spectrum Disorder? J Physiol (Paris) 2016; 110 (4): 434-438.

6. Carmassi C, Palagini L, Caruso D, et al. Systematic Review of Sleep Disturbances and Circadian Sleep Desynchronization in Autism Spectrum Disorder: Toward an Integrative Model of a Self-Reinforcing Loop. Front Psychiatry 2019; 10: 366.

7. Tudor ME, Hoffman CD, Sweeney DP. Children With Autism: Sleep Problems and Symptom Severity. Focus Autism Dev Disabil 2012; 27 (4): 254-262.

8. Zisapel N. New perspectives on the role of melatonin in human sleep, circadian rhythms and their regulation. Br J Pharmacol 2018; 175 (16): 3190-3199.

9. Posadzki PP, Bajpai R, Kyaw BM, et al. Melatonin and health: an umbrella review of health outcomes and biological mechanisms of action. BMC Med 2018; 16 (1): 18.

10. Gagnon K, Godbout R. Melatonin and Comorbidities in Children with Autism Spectrum Disorder. Curr Dev Disord Rep 2018; 5 (3): 197-206.

11. Praninskienė R, Dumalakienė I, Kemežys R, Mauricas M, Jučaitė A. Diurnal Melatonin Patterns in Children: Ready to Apply in Clinical Practice? Pediatr Neurol 2012; 46 (2): 70-76.

12. Arendt J. Melatonin and the pineal gland: influence on mammalian seasonal and circadian physiology. Rev Reprod 1998; 3 (1): 13-22.

13. Lord C, Rutter M, DiLavore PC, Risi S, Gotham K, Bishop S. Autism diagnostic observation schedule. 2012.

14. Lord C, Pickles A, McLennan J, et al. Diagnosing autism: analyses of data from the Autism Diagnostic Interview. J Autism Dev Disord 1997; 27 (5): 501-517.

15. Gotham K, Pickles A, Lord C. Standardizing ADOS Scores for a Measure of Severity in Autism Spectrum Disorders. J Autism Dev Disord 2009; 39 (5): 693-705.

16. Owens JA, Spirito A, McGuinn M. The Children's Sleep Habits Questionnaire (CSHQ): psychometric properties of a survey instrument for school-aged children. Sleep 2000; 23 (8): 1043-1051.

17. Aldhous ME, Arendt J. Radioimmunoassay for 6-Sulphatoxymelatonin in Urine Using an Iodinated Tracer. Ann Clin Biochem Int J Biochem Lab Med 1988; 25 (3): 298-303. 
18. Tang KWA, Toh QC, Teo BW. Normalisation of urinary biomarkers to creatinine for clinical practice and research - when and why. Singapore Med J 2015; 56 (1): 7-10.

19. Elrod MG, Hood BS. Sleep Differences Among Children With Autism Spectrum Disorders and Typically Developing Peers: A Meta-analysis. J Dev Behav Pediatr 2015; 36 (3): 166-177.

20. Souders MC, Zavodny S, Eriksen W, et al. Sleep in Children with Autism Spectrum Disorder. Curr Psychiatry Rep 2017; 19 (6).

21. Kulman G, Lissoni P, Rovelli F, Roselli MG, Brivio F, Sequeri P. Evidence of pineal endocrine hypofunction in autistic children. Neuro Endocrinol Lett 2000; 21 (1): 31-34.

22. Nir I, Meir D, Zilber N, Knobler H, Hadjez J, Lerner Y. Brief report: Circadian melatonin, thyroid-stimulating hormone, prolactin, and cortisol levels in serum of young adults with autism. J Autism Dev Disord 1995; 25 (6): 641-654.

23. Tordjman S, Anderson GM, Pichard N, Charbuy H, Touitou Y. Nocturnal excretion of 6-sulphatoxymelatonin in children and adolescents with autistic disorder. Biol Psychiatry 2005; 57 (2): 134-138.
24. Tordjman S, Anderson GM, Bellissant E, et al. Day and nighttime excretion of 6-sulphatoxymelatonin in adolescents and young adults with autistic disorder. Psychoneuroendocrinology 2012; 37 (12): 1990-1997.

25. Leu RM, Beyderman L, Botzolakis EJ, Surdyka K, Wang L, Malow BA. Relation of Melatonin to Sleep Architecture in Children with Autism. J Autism Dev Disord 2011; 41 (4): 427-433.

26. Braam W, Ehrhart F, Maas APHM, Smits MG, Curfs L. Low maternal melatonin level increases autism spectrum disorder risk in children. Res Dev Disabil 2018; 82: 79-89.

27. Stefanovic B, Spasojevic N, Jovanovic P, Ferizovic H, Dronjak S. Melatonin modulate the expression of $\alpha 1$ - and $\beta 2$-adrenoceptors in the hippocampus of rats subjected to unpredictable chronic mild stress. Bratisl Med J 2018; 119 (7): 429-433.

28. Checa-Ros A, Muñoz-Hoyos A, Molina-Carballo A, et al. Analysis of Different Melatonin Secretion Patterns in Children With Sleep Disorders: Melatonin Secretion Patterns in Children. J Child Neurol 2017; 32 (12): 1000-1008. 